\title{
Renal sympathetic denervation increases renal
} blood volume per cardiac cycle: a serial magnetic resonance imaging study in resistant hypertension

This article was published in the following Dove Press journal: International Journal of Nephrology and Renovascular Disease 3I August 2017

Number of times this article has been viewed

\author{
Sinny Delacroix ${ }^{1,2}$ \\ Ramesh G Chokka ${ }^{1,3}$ \\ Adam J Nelson ${ }^{1,2}$ \\ Dennis T Wong ${ }^{4}$ \\ Samuel Sidharta ${ }^{1,2}$ \\ Stephen M Pederson ${ }^{5}$ \\ Adil Rajwani ${ }^{1,2}$ \\ Joanne Nimmo ${ }^{1,2}$ \\ Karen S Teo ${ }^{1,2}$ \\ Stephen G Worthley ${ }^{1,2}$ \\ 'Cardiovascular Research Centre, \\ Royal Adelaide Hospital, ${ }^{2}$ Department \\ of Medicine, University of Adelaide, \\ Adelaide, SA, ${ }^{3}$ South Australian Health \\ and Medical Research Institute, \\ University of Adelaide, Adelaide, SA, \\ ${ }^{4}$ Monash Medical Centre, Clayton, \\ VIC, ${ }^{5}$ Bioinformatics Hub, School \\ of Biological Sciences, University of \\ Adelaide, Adelaide, SA, Australia
}

Correspondence: Sinny Delacroix Department of Cardiovascular Medicine, Level 3 Res. Wing MDP I7,

North Terrace Adelaide, SA 5000, Australia

Tel $+6 \mid 882225608$

Fax +6I 882222454

Email sinny.delacroix@adelaide.edu.au
Aim: Preclinical studies have demonstrated improvements in renal blood flow after renal sympathetic denervation (RSDN); however, such effects are yet to be confirmed in patients with resistant hypertension. Herein, we assessed the effects of RSDN on renal artery blood flow and diameter at multiple time points post-RSDN.

Methods and results: Patients $(n=11)$ with systolic blood pressures $\geq 160 \mathrm{mmHg}$ despite taking three or more antihypertensive medications at maximum tolerated dose were recruited into this single-center, prospective, non-blinded study. Magnetic resonance imaging indices included renal blood flow and renal artery diameters at baseline, 1 month and 6 months. In addition to significant decreases in blood pressures $(p<0.0001)$, total volume of blood flow per cardiac cycle increased by $20 \%$ from $6.9 \pm 2 \mathrm{~mL}$ at baseline to $8.4 \pm 2 \mathrm{~mL}(p=0.003)$ at 1 month and to $8.0 \pm 2 \mathrm{~mL}(p=0.04) 6$ months post-procedure, with no changes in the renal blood flow. There was a significant decrease in renal artery diameters from $7 \pm 2 \mathrm{~mm}$ at baseline to $6 \pm 1 \mathrm{~mm}(p=0.03)$ at 1 month post-procedure. This decrease was associated with increases in maximum velocity of blood flow from $73 \pm 20 \mathrm{~cm} / \mathrm{s}$ at baseline to $78 \pm 19 \mathrm{~cm} / \mathrm{s}$ at 1 month post-procedure. Notably, both parameters reverted to $7 \pm 2 \mathrm{~mm}$ and $72 \pm 18 \mathrm{~cm} / \mathrm{s}$, respectively, 6 months after procedure. Conclusion: RSDN improves renal physiology as evidenced by significant improvements in total volume of blood flow per cardiac cycle. Additionally, for the first time, we identified a transient decrease in renal artery diameters immediately after procedure potentially caused by edema and inflammation that reverted to baseline values 6 months post-procedure.

Keywords: renal denervation, resistant hypertension, magnetic resonance imaging, renal blood flow, renal physiology

\section{Introduction}

HTN affects over one billion people worldwide and accounts for at least $45 \%$ of deaths due to heart disease and $51 \%$ of deaths due to stroke. ${ }^{1}$ The high morbidity and mortality rates associated with the pathology are often the consequence of poorly managed HTN, resulting in cardiac hypertrophy, heart failure, stroke and chronic kidney disease. Treatment of the disease remains challenging despite the availability of a plethora of pharmaceutical agents because the etiopathophysiology of the disease is not evident in most patients limiting an individualized preventive and therapeutic approach that is further compounded by patient incompliance.

The idea that the kidneys play a pivotal role in the development and maintenance of HTN began in the 19th century when it was hypothesized that changes in urine production caused increased vascular resistance resulting in increased blood pressures. $^{2,3}$ However, whether renal dysfunction is the cause or the effect of HTN is 
still debatable. To this extent, Guyton et al proposed that extracellular fluid volume is maintained by the kidneys via renal excretion of sodium and water depending on dietary intake. $^{5-7}$ The kidney in response to increased renal perfusion pressures alters sodium and water excretion to maintain fluid homeostasis, whereby increased pressure translates to increased sodium and water excretion which is now termed as "pressure natriuresis". In normotensive individuals, renal pressure natriuresis is capable of handling transient variations of intravascular volumes triggered by increased heart rate or increased peripheral vascular resistance. However, it is postulated that with consistent increases in pressures, the kidney shifts to a new higher-than-normal equilibrium point for salt and water excretion resulting in higher blood pressures. $^{4-7}$ Several renal transplantation studies ${ }^{8-12}$ have revealed that HTN follows the kidneys; the first study demonstrated that transplantation of kidneys from hypertensive rats to normotensive rats resulted in HTN in the normal mice and similarly transplanting kidneys from normotensive rats to hypertensive rats resulted in abrogation of blood pressures in the hypertensive rats, revealing that the kidney might play an integral role in the development of HTN.

RSDN is a treatment option in patients with resistant HTN who are unable to attain goal blood pressures despite treatment with three or more antihypertensive medications including a diuretic. Several trials have demonstrated that, in carefully selected patients, the procedure decreases both office blood pressure and ABP. That being said, the recent Symplicity HTN-3 study showed that there were no significant differences in blood pressure decreases between the treatment arm and the sham group. ${ }^{13}$ A noteworthy limitation of this treatment option is the absence of an objective measure of procedural success and efficacy besides blood pressure reduction. Therefore, we investigated the potential of MRI in identifying changes in renal blood flow and renal artery diameters as measures of change in renal physiology post-RSDN.

\section{Methods}

\section{Aim and study design}

This is a prospective single-center, single-arm study designed to assess the effects of RSDN on renal physiology (ClinicalTrials.gov trial ID: NCT02164435, registration date: 12/06/2014).

\section{Study population}

The Royal Adelaide Hospital ethics committee approved the study, and patients provided written informed consent. Subjects capable of providing informed consent who were $\geq 18$ years of age, with an office $\mathrm{SBP} \geq 160 \mathrm{mmHg}$ (and subjects with diabetes mellitus with an office SBP $\geq 150 \mathrm{mmHg}$ ) within 14 days of the procedure, and who were on three or more antihypertensive medications including a diuretic concurrently at maximum tolerated doses were enrolled in the study. Subjects with documented intolerance precluding them from taking standard antihypertensives were also included. Subjects who met the following standard exclusion criteria for RSDN were excluded from study participation: presence of significant renovascular abnormalities like renal artery stenosis or renal arteries with diameter(s) $<4 \mathrm{~mm}$, presence of secondary HTN, eGFR $<45$ $\mathrm{mL} / \mathrm{min} / 1.73 \mathrm{~m}^{2}$ as calculated by the Modification of Diet in Renal Disease formula, hemodynamically significant valvular heart disease and presence of implants or claustrophobia preventing CMR imaging. Blood pressures were measured as per Joint National Committee VII guidelines after 5-minute rest in a seated position with an automatic OMRON T9P monitor (Omron Healthcare, Venon Hills, IL, USA). ${ }^{14}$ An average of three readings was taken for the purposes of this study.

Blood and urine were collected for complete blood count, basic metabolic profile, serum creatinine, eGFR and for urine albumin-to-creatinine ratio, and the medications were also reviewed. Eligible subjects were scheduled for RSDN using the EnligHTN"M Renal Denervation System (St. Jude Medical).

\section{Medication}

There were no medication changes in any of the patients during the course of the study.

\section{Magnetic resonance imaging}

Phase-contrast velocity mapping was used to assess the velocity, volume and pattern of renal blood flow. Velocityencoded sequences are able to measure the phase shifts of moving nuclei compared with stationary nuclei with the extent of the shift being proportional to the flow velocity. A second data set was acquired with an inverted gradient and subtracted from the first in order to eliminate phase shifts caused by factors other than blood flow. Phase-contrast MRI of the renal arteries was obtained without a breath hold as the venous flow pattern can change significantly with breath holding. No contrast was required for this sequence. Analysis was performed using commercially available semiautomated quantitative software on Cvi42 version 5.3.2.

\section{Statistical analysis}

Statistical comparisons were performed using paired $t$-tests with the exception of eGFR which was analyzed using the 
nonparametric Wilcoxon signed rank test in order to account for clear violations of normality. Results are expressed as mean $\pm \mathrm{SD}$, and $p<0.05$ was considered statistically significant.

\section{Results}

\section{Study population}

A total of 14 patients were enrolled in the study, and 13 proceeded to RSDN. In one patient, the procedure was terminated due to severe iliofemoral artery tortuosity and another patient was diagnosed with hypertrophic cardiomyopathy and was therefore excluded from the study. The baseline patient characteristics are presented in Table 1.

Table I Baseline patient characteristics

\begin{tabular}{ll}
\hline Parameters $(\mathbf{n}=\mathrm{I} \mathbf{I})$ & Mean \pm SD or $\mathbf{n}(\%)$ \\
\hline Age (years) & $60 \pm 12$ \\
Male gender & $7(64)$ \\
BMI $\left(\mathrm{kg} / \mathrm{m}^{2}\right)$ & $31 \pm 6$ \\
Type 2 diabetes & $3(27)$ \\
Hypercholesterolemia & $4(36)$ \\
Medications & \\
Number of antihypertensives & $5.2 \pm 1 . \mathrm{I}$ \\
ACE inhibitors/ARBs & $11(100)$ \\
Beta blockers & $9(82)$ \\
Alpha blockers & $7(64)$ \\
Sympatholytics & $6(55)$ \\
Calcium channel blockers & $8(73)$ \\
Diuretics & $11(100)$ \\
Systolic blood pressure (mmHg) & $181 \pm 19$ \\
Diastolic blood pressure $(\mathrm{mmHg})$ & $100 \pm 16$ \\
Pulse pressure (mmHg) & $82 \pm 17$ \\
\hline
\end{tabular}

Abbreviations: $\mathrm{BMI}$, body mass index; ACE, angiotensin-converting enzyme; ARBs, angiotensin receptor blockers.

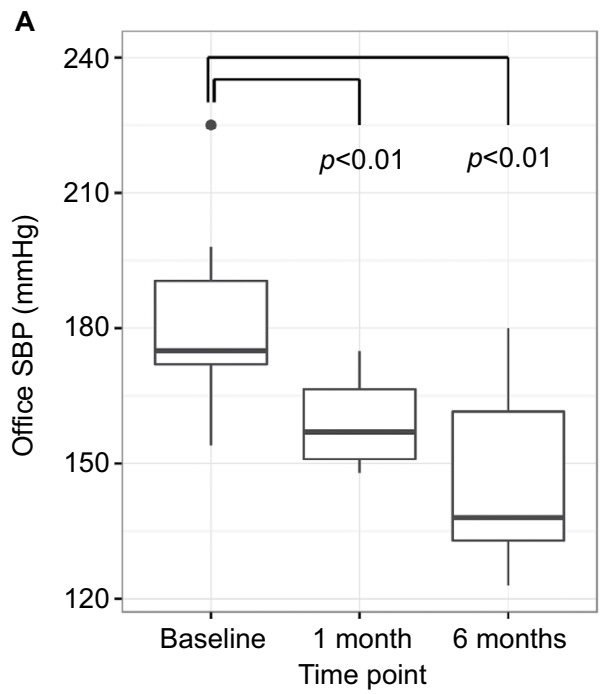

\section{Changes in blood pressure and heart rate}

There was a significant reduction in mean SBP/DBP postRSDN which decreased from $181 \pm 19 / 100 \pm 16 \mathrm{mmHg}$ at baseline to $160 \pm 9 / 93 \pm 13 \mathrm{mmHg}$ at 1 month $(\mathrm{n}=11, p<0.014)$ and $147 \pm 19 / 85 \pm 17 \mathrm{mmHg} 6$ months after $\mathrm{RSDN}(\mathrm{n}=11$, $p<0.0001$; Figure 1A and B). ABPs were also measured in these patients, and while there was no change in mean ABPs, i.e., $147 / 83 \pm 21 / 16 \mathrm{mmHg}$ at baseline and 141/78 $\pm 12 / 12$ $\mathrm{mmHg}$ at 1 month and 147/83 $\pm 15 / 15 \mathrm{mmHg}$ at 6 months post-procedure (Figure S1A and B), it was noted that patients with higher baseline ABPs had a reduction post-procedure $(p<0.005)$. There was a statistically significant decrease in mean heart rate from $80.2 \pm 15 \mathrm{bpm}$ to $70.9 \pm 12 \mathrm{bpm}$ at 1 month but only a numerical decrease at 6 months at which time the mean heart rate was $75.5 \pm 14 \mathrm{bpm}$ (Figure S1C).

In almost all RSDN studies, there has been a clear group of poor responders to treatment; therefore, albeit being a small study, patients were grouped into good, inconsistent and poor responders based on any decrease in OSBP, ODBP and each of the hormone/protein measurements or any increase in renal blood flow $(\mathrm{RBF})_{-} \mathrm{Vol} / \mathrm{min}, \mathrm{RBF} \_\mathrm{TV}, \mathrm{RBF} \_\mathrm{Max} V \mathrm{l}$, renal artery diameter and eGFR.

$\underline{\text { Table S1 demonstrates the classification, and Figure S2 }}$ shows the heat map of changes in these variables at 1 month and 6 months post-RSDN.

The $95 \%$ confidence intervals for percentage change of office blood pressure in the good, inconsistent and poor responders are shown in Figure S3.

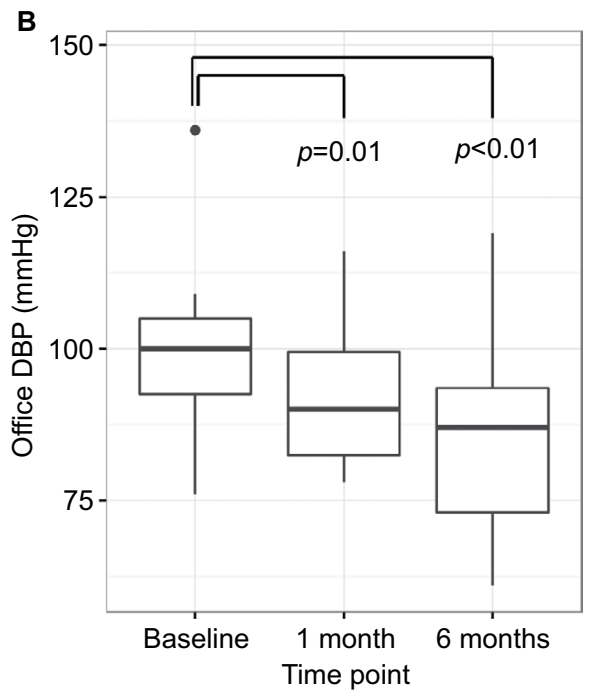

Figure I Blood pressure post-renal sympathetic denervation ( $n=1$ I). Significant reduction in office systolic (A) and diastolic (B) blood pressures. Abbreviations: SBP, systolic blood pressure; DBP, diastolic blood pressure. 
A

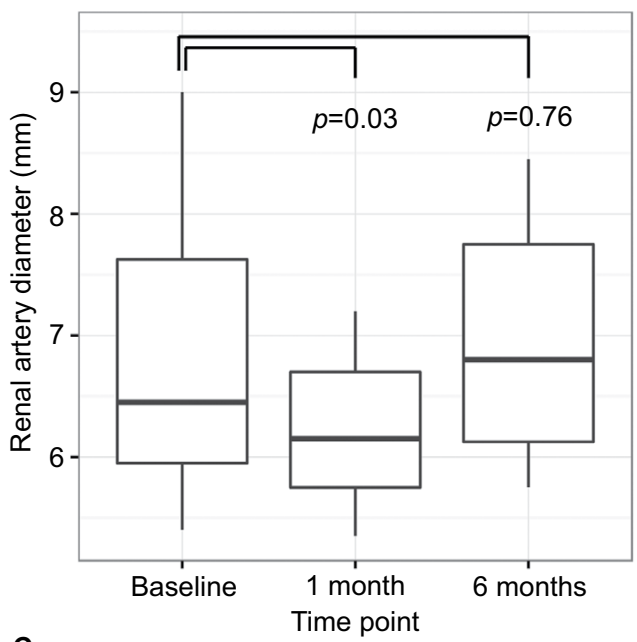

C

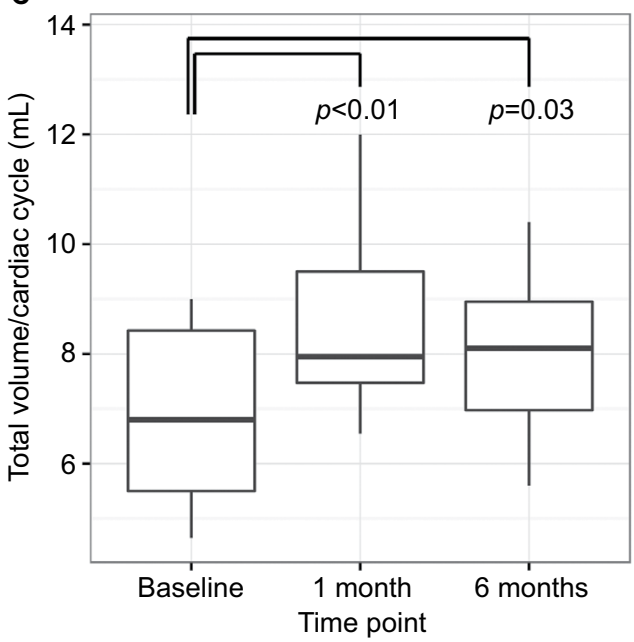

B

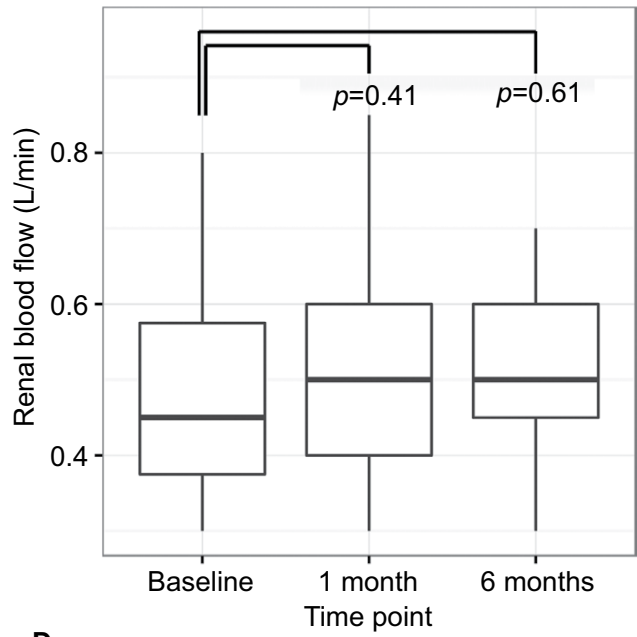

D

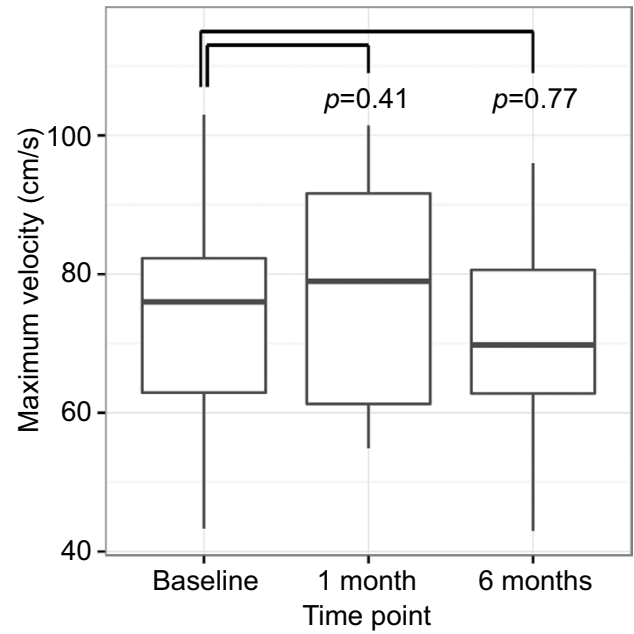

Figure 2 Effects of renal denervation on renal physiology $(n=I I)$. Renal artery diameter reductions at I month $(p=0.03)$ returned to baseline values at 6 months $(p=0.76)$ (A). No change was noted in renal blood flows (B). Total volume per cardiac cycle improved from baseline at both I month ( $p=0.003)$ and 6 months $(p=0.03)(\mathbf{C})$. No change was noted in maximum velocity per cardiac cycle $(p>0.4)(\mathbf{D})$.

\section{Effect of RSDN on renal anatomy and physiology}

There was a significant reduction in renal artery diameters from $7 \pm 2 \mathrm{~mm}$ at baseline to $6 \pm 1 \mathrm{~mm}$ at 1 month after RSDN $(\mathrm{n}=11, p=0.03)$. However, this was noted to revert to baseline values of $7 \pm 2 \mathrm{~mm}$ at 6 months post-procedure with no significant difference detected ( $\mathrm{n}=11, p=0.76$; Figure 2A). Direct comparison between time points further confirmed the significance of this reversion $(\mathrm{n}=11, p=0.04)$. There was no change in renal blood flow, which remained near $0.5 \pm 0.2 \mathrm{~L} / \mathrm{min}$ at both 1 month $(\mathrm{n}=11, p=0.41)$ and 6 months $(\mathrm{n}=11, p=0.61)$ (Figure 2B). Conversely, there was a significant improvement in total volume per cardiac cycle from $6.9 \pm 2 \mathrm{~mL}$ at baseline to $8.4 \pm 2 \mathrm{~mL}$ at 1 month $(\mathrm{n}=11, p=0.003)$ and $8 \pm 2 \mathrm{~mL}$ at 6 months $(\mathrm{n}=11, p=0.04)$ (Figure $2 \mathrm{C}$ ). The maximum velocities in the renal arteries were $73 \pm 20 \mathrm{~cm} / \mathrm{s}$ at baseline compared to $78 \pm 19 \mathrm{~cm} / \mathrm{s}$ at 1 month $(\mathrm{n}=11, p=0.41)$ and $72 \pm 18 \mathrm{~cm} / \mathrm{s}$ at 6 months post-RSDN ( $\mathrm{n}=11, p=0.77$ ) (Figure 2D). The 95\% confidence intervals for percentage change in the aforementioned parameters are shown in Figure S4.

\section{Renal function post-RSDN}

There was a significant improvement in eGFR from $67 \mathrm{~mL} /$ $\mathrm{min} / 1.73 \mathrm{~m}^{2}$ to $82 \mathrm{~mL} / \mathrm{min} / 1.73 \mathrm{~m}^{2} 6$ months after procedure $(\mathrm{n}=10, p=0.02)$ (Figure 3A). Although mean serum aldosterone levels decreased numerically from $560 \mathrm{pmol} / \mathrm{L}$ to $370 \mathrm{pmol} / \mathrm{L}(\mathrm{n}=9, p=0.85)$, this is of limited, if any, clinical significance considering the outliers (Figure 3B). That being said, patients with higher baseline values showed pronounced improvement. While not statistically significant, there was an $8 \%$ decrease in plasma creatinine from $77 \mu \mathrm{mol} / \mathrm{L}$ to 73 $\mu \mathrm{mol} / \mathrm{L}$ and $71 \mu \mathrm{mol} / \mathrm{L}$ at baseline, 1 month and 6 months, 
A

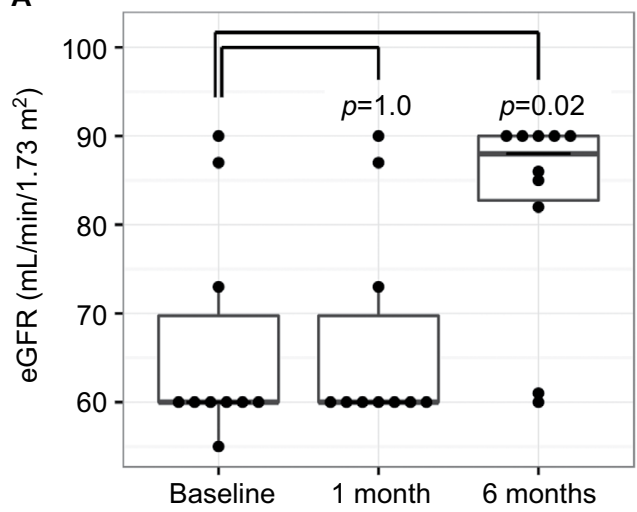

C

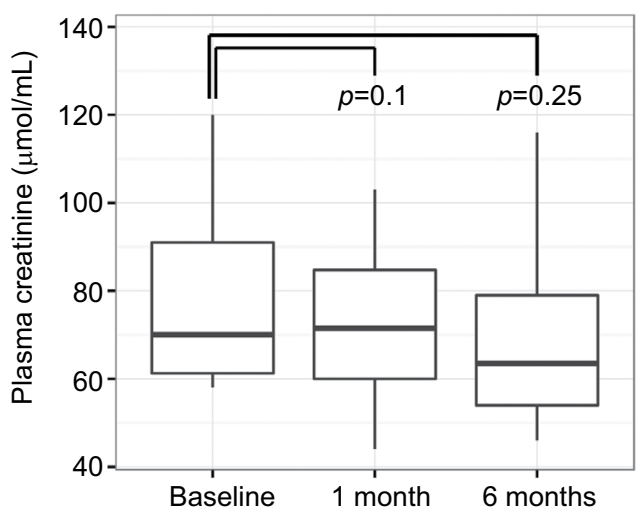

E

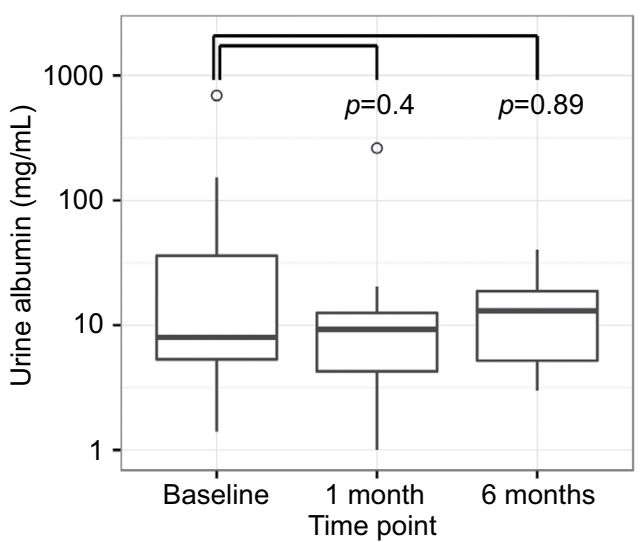

B

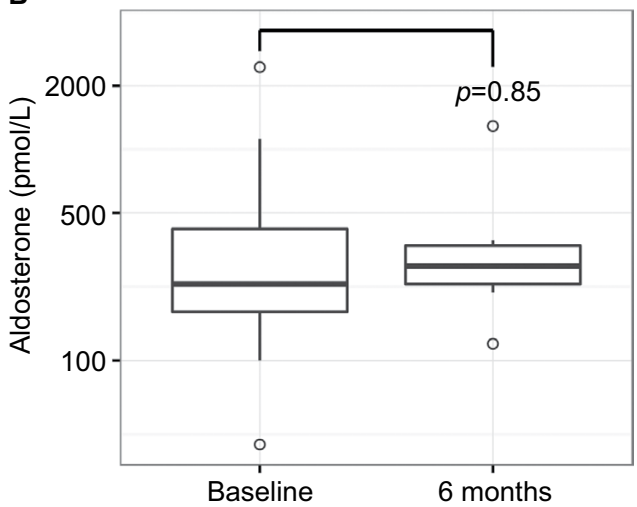

D

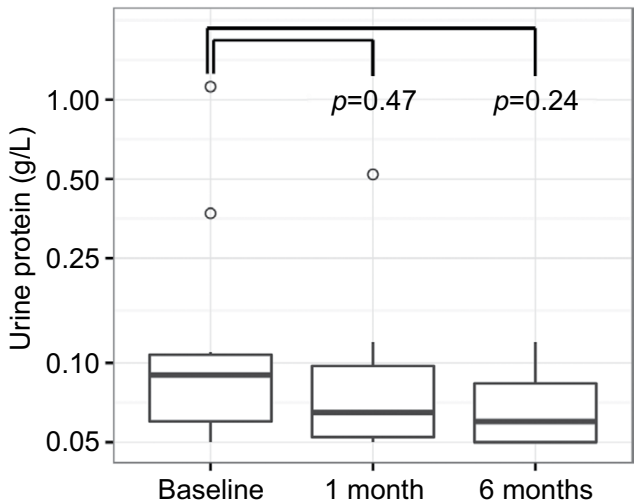

F

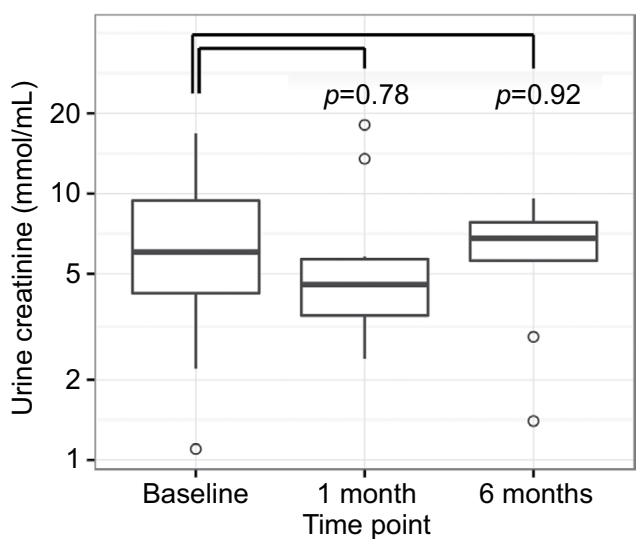

Figure 3 Renal denervation in the context of kidney function. Renal denervation significantly improved eGFR at 6 months ( $p=0.02$, $n=10)(\mathbf{A})$. All data points are shown as an overlay to the box plot using filled circles. Also, the changes in plasma aldosterone $(p=0.85, n=9)(B)$, plasma creatinine $(p=0.1$ and $0.25, n=10)(C)$, urine protein $(p=0.47$ and 0.24$)(\mathbf{D})$, urine albumin $(p=0.4$ and 0.89$)(\mathbf{E})$, and urine creatinine $(p=0.78$ and $0.92, n=10)(\mathbf{F})$ are shown. For plots $(\mathbf{B})-(\mathbf{F})$, outlier points are shown as empty circles. Abbreviation: eGFR, estimated glomerular filtration rate.

respectively ( $n=10, p \geq 0.1$; Figure $3 C$ ). Similarly, there were no statistically significant changes in urinary protein concentrations or urine creatinine level $(n=10, p>0.05$; Figure 3D-F). Log scales were used for these parameters for $95 \%$ confidence intervals for percentage change due to the variability seen in these measurements and are demonstrated in Figure S5.

\section{Discussion}

Human and animal studies have demonstrated the role of sympathetic nervous system in the development and maintenance of HTN making RSDN an optimal choice for patients nonresponsive to medical therapies. ${ }^{15}$ That being said, the effects of this procedure on renal physiology are largely debated, and there are limited data that assess renal 
outcomes post-RSDN. Studies by Hering et al, ${ }^{16}$ Ott et al ${ }^{17}$ and Zhang et $\mathrm{al}^{18}$ have demonstrated the safety and potentially beneficial outcomes in patients with stage 3-4 chronic kidney disease and resistant HTN. Similarly, a study by Ott et al has shown that renal perfusion and renal vascular resistance was unaltered post-procedure; ${ }^{19}$ however, to our knowledge, this is the first study utilizing phase-contrast velocity mapping to determine renal effects of the procedure alongside eGFR, the widely accepted parameter indicative of renal function.

Catheter-based RSDN involves ablation of nerves within the adventitia of the renal arteries, and the prospective of renal artery stenosis post-procedure has been long contested. Herein, alongside a drop in SBP and DBP, there was a significant decrease in renal artery diameters 1 month post-procedure, which returned to baseline levels at 6 months post-procedure. The latter was associated with an increase in velocity of renal blood flow. The transient nature of these changes could be attributed to immediate post-ablative reactive processes such as inflammation and repair, which are temporary and eventually resolve as evidenced here. Consistent with previous reports demonstrating decreased renal vascular resistance, this study shows an improvement in total volume of blood to the kidney per cardiac cycle. ${ }^{19}$ This could be especially important in patients demonstrating end organ damage as a consequence of consistently increased blood pressures.

There is enough evidence to indicate that RSDN results in decreased renal epinephrine spillover, ${ }^{20}$ but assessment of renal function as gauged by plasma aldosterone and urine biochemistry such as urine albumin and creatinine remains sparse. This study demonstrates that RSDN resulted in $22 \%$ improvement in eGFR at 6 months post-procedure. Moreover, although not statistically significant, there was an $8 \%$ improvement in plasma creatinine with a drop in plasma aldosterone levels in patients with higher baseline values and a $16 \%$ numerical increase in urine creatinine. While it is challenging to interpret these results owing to the limited numbers, the data suggest that the improvement in total volume of blood flow per cardiac cycle post-RDN is probably not due to local vascular resistance but rather due to a more effective and efficient kidney resulting from decreased blood pressures that potentially reset the renal equilibrium. Furthermore, a decreased heart rate possibly permits longer transition times for blood flow through the kidneys enabling longer and better filtration. Similarly, this could well be attributed to expansion of venous capacitance that likely occurs with reductions in sympathetic nervous system activity leading to greater intravascular volume and loading of the ventricle. At this point, these are merely hypotheses, and larger targeted studies are required to demonstrate the mechanism of these changes. A Pearson correlation (Figure S6) was used to tease out any correlations, and although we did find some consistent correlations, as expected with small patient numbers, the effects were not overwhelming and failed to provide any clear explanation for the mechanism of the renal effects seen post-RSDN. That being said, it can be postulated that these results insinuate that the procedure might help prevent deterioration of renal function in these chronically ill patients with questionable renal physiology.

Chronic kidney disease often accompanies resistant HTN. While it is challenging to determine if the above effects of RSDN on renal physiology are due to decreased blood pressure or due to alternative mechanisms resulting from decreased sympathetic drive, they warrant further investigation. This study identifies that RSDN is safe and that it might potentially be beneficial; however, the significance of these findings remains to be established in larger studies.

\section{Limitations}

First, the sample size is small, but the study has identified significant improvements in parameters such as eGFR, which are routinely used to assess renal function. Second, the end points were not performed by blinded investigators. Finally, absence of a control arm makes interpretation of results challenging; however, the data presented corroborate previous studies and assess the effects of RSDN from an MRI-based renal perspective. Similarly, it should be noted that although each patient is his own control, since we do not have a control group per se, regression to the mean might overestimate blood pressure reductions post-renal denervation.

\section{Abbreviations}

ABP, ambulatory blood pressure

$\mathrm{CMR}$, cardiac magnetic resonance

DBP, diastolic blood pressure

eGFR, estimated glomerular filtration rate

HTN, hypertension

ODBP, office diastolic blood pressure

OSBP, office systolic blood pressure

MRI, magnetic resonance imaging

RSDN, renal sympathetic denervation

SBP, systolic blood pressure

\section{Acknowledgments}

The authors would like to acknowledge the technical CMR expertise provided by Ms. Kerry Williams, without whom this project would not have been possible. The abstract of 
this article has been published in the Circulation journal (2014;130:A18099).

\section{Disclosure}

SGW received honoraria and grant support from St. Jude Medical and Medtronic. JN is an employee of St. Jude Medical. All other authors have no conflicts of interest to disclose in this work.

\section{References}

1. Lawes CM, Vander Hoorn S, Rodgers A; International Society of Hypertension. Global burden of blood-pressure-related disease, 2001. Lancet. 2008;371(9623):1513-1518.

2. Goldblatt H, Lynch J, Hanzal RF, Summerville WW. Studies on experimental hypertension I. The production of persistent elevation of systolic blood pressure by means of renal ischemia. J Exp Med. 1934;59(3):347-379.

3. Bright R. Tabular view of the morbid appearances in 100 cases connected with albuminous urine. Guy's Hosp Rep. 1836;1:380.

4. Aperia AC, Broberge CG, Soderlund S. Relationship between renal artery perfusion pressure and tubular sodium reabsorption. Am J Physiol. 1971;220:1205-1212.

5. Guyton AC. Blood pressure control--special role of the kidneys and body fluids. Science. 1991;252(5014):1813-1816.

6. Guyton AC, Coleman TG, Cowley AW Jr, Liard JF, Norman RA Jr, Manning RD Jr. Systems analysis of arterial pressure regulation and hypertension. Ann Biomed Eng. 1972;1(2):254-281.

7. Guyton AC, Scheel KW, Coleman TG, Cowley AW, Manning RD, Norman RA. Arterial pressure regulation: overriding dominance of the kidneys in long-term regulation and in hypertension. Am J Med. 1972;52(5):584-594.

8. Bianchi G, Fox U, Di Francesco F, Giovanetti AM, Pagetti D. Blood pressure changes produced by kidney cross-transplantation between spontaneously hypertensive rats and normotensive rats. Clin Sci Mol Med. 1974;47(5):435-448.

9. Curtis JJ, Luke RG, Dustan HP, et al. Remission of essential hypertension after renal transplantation. N Engl J Med. 1983;309(17):1009-1015.
10. Dahl LK, Heine M. Primary role of renal homografts in setting chronic blood pressure levels in rats. Circ Res. 1975;36(6):692-696.

11. Dahl LK, Heine M, Thompson K. Genetic influence of kidneys on blood pressure. Evidence from chronic renal homografts in rats with opposite predispositions to hypertension. Circ Res. 1974;34(1):94-101.

12. Kawabe K, Watanabe TX, Shiono K, Sokabe H. Influence on blood pressure of renal isografts between spontaneously hypertensive and normotensive rats, utilizing the F1 hybrids. Jpn Heart J. 1978;19(6): 886-894.

13. Bhatt DL, Kandzari DE, O'Neill WW, et al; SYMPLICITY HTN-3 Investigators. A controlled trial of renal denervation for resistant hypertension. N Engl J Med. 2014;370(15):1393-1401.

14. Chobanian AV, Bakris GL, Black HR, et al; National Heart, Lung, and Blood Institute Joint National Committee on Prevention, Detection, Evaluation, and Treatment of High Blood Pressure; National High Blood Pressure Education Program Coordinating Committee. The Seventh Report of the Joint National Committee on Prevention, Detection, Evaluation, and Treatment of High Blood Pressure: the JNC 7 report. J Am Med Assoc. 2003;289(19):2560-2572.

15. Chokka RG, Delacroix S, Psaltis PJ, Anavekar NS, Worthley SG. Percutaneous renal denervation and the second generation EnligHTN System. Minerva Cardioangiol. 2014;62(1):99-104.

16. Hering D, Mahfoud F, Walton AS, et al. Renal denervation in moderate to severe CKD. J Am Soc Nephrol. 2012;23(7):1250-1257.

17. Ott C, Mahfoud F, Schmid A, et al. Renal denervation preserves renal function in patients with chronic kidney disease and resistant hypertension. J Hypertens. 2015;33(6):1261-1266.

18. Zhang ZH, Yang K, Jiang F-L, Zeng L-X, Jiang W-H, Wang X-Y. The effects of catheter-based radiofrequency renal denervation on renal function and renal artery structure in patients with resistant hypertension. J Clin Hypertens (Greenwich). 2014;16(8):599-605.

19. Ott C, Janka R, Schmid A, et al. Vascular and renal hemodynamic changes after renal denervation. Clin J Am Soc Nephrol. 2013;8(7):1195-1201.

20. Symplicity HTN-2 Investigators, Esler MD, Krum H, et al. Renal sympathetic denervation in patients with treatment-resistant hypertension (The Symplicity HTN-2 Trial): a randomised controlled trial. Lancet. 2010;376(9756):1903-1909.
The International Journal of Nephrology and Renovascular Disease is an international, peer-reviewed open access journal focusing on the pathophysiology of the kidney and vascular supply. Epidemiology, screening, diagnosis, and treatment interventions are covered as well as basic science, biochemical and immunological studies. The manuscript management system is completely online and includes a very quick and fair peer-review system, which is all easy to use. Visit http://www. dovepress.com/testimonials.php to read real quotes from published authors. 